$11-1998$

\title{
Semiclassical Representation of Width-Weighted Spectra
}

M. W. Beims

V. Kondratovich

William \& Mary

John B. Delos

William \& Mary, jbdelos@wm.edu

Follow this and additional works at: https://scholarworks.wm.edu/aspubs

Part of the Physics Commons

\section{Recommended Citation}

Beims, M. W.; Kondratovich, V.; and Delos, John B., Semiclassical Representation of Width-Weighted Spectra (1998). Physical Review Letters, 81(21), 4537-4540.

https://doi.org/10.1103/PhysRevLett.81.4537

This Article is brought to you for free and open access by the Arts and Sciences at W\&M ScholarWorks. It has been accepted for inclusion in Arts \& Sciences Articles by an authorized administrator of W\&M ScholarWorks. For more information, please contact scholarworks@wm.edu. 


\title{
Semiclassical Representation of Width-Weighted Spectra
}

\author{
M. W. Beims, V. Kondratovich, and J. B. Delos \\ Department of Physics, College of William and Mary, Williamsburg, Virginia 23187
}

(Received 29 June 1998)

\begin{abstract}
We consider a system having decaying states with complex energies $E_{j}-i \Gamma_{j} / 2$, and we define the "width-weighted spectrum" as $D \Gamma(E)=\sum_{j} \Gamma_{j} \delta\left(E-E_{j}\right)$. We derive a semiclassical formula for this width-weighted spectrum, a formula which is analogous to the periodic-orbit representation of the density of states. The formula applies if classical motion is regular and if decay occurs by tunneling through a barrier. The semiclassical formula involves not periodic orbits or closed orbits, but action integrals associated with irreducible loops on the "extremal torus," on which the particle climbs up the barrier and hangs at the top. Calculations confirm the validity of the formula. [S0031-9007(98)07690-X]

PACS numbers: 03.65.Sq
\end{abstract}

Gutzwiller [1] gave a semiclassical representation of the density of states of a quantum system, $\rho(E)=$ $\sum_{j} \delta\left(E-E_{j}\right)$, in terms of a sum over periodic orbits of the corresponding classical system. An alternative derivation of the relationship between the quantum density of states and classical periodic orbits, suitable for integrable systems with regular spectra, was given by Berry and Tabor [2]. Later, Du and Delos [3] gave a semiclassical formula for the photoabsorption spectrum of an atom, the oscillator-strength density $D f(E)=$ $\sum_{j} f_{j} \delta\left(E-E_{j}\right)$, where $f_{j}$ is the oscillator strength for transition from a low-lying initial state into state $j$. In this case the semiclassical representation involves a sum over closed classical orbits of the electron which start from the nucleus and return to it. Recently, Creagh and Whelan [4] considered a splitting-weighted density of states $D \Delta(E)=\sum_{j} \Delta E_{j} \delta\left(E-E_{j}\right)$, where $\Delta E_{j}$ is the small energy gap between symmetric and antisymmetric states in a model double-well system. Their semiclassical representation of this quantity involves a few real periodic orbits in a chaotic region of phase space, as well as complex orbits to describe the underbarrier motion.

In this Letter we consider a system having quasibound states with complex energies $\mathcal{E}_{j}=E_{j}-i \Gamma_{j} / 2$, and we define the "width-weighted spectrum" as

$$
D \Gamma(E)=\sum_{j} \Gamma_{j} \delta\left(E-E_{j}\right) .
$$

Each quasibound state with real energy $E=E_{j}$ is weighted by the width $\Gamma_{j}$, which is related to the decay time of the state by $\tau_{j}=\hbar / \Gamma_{j}$. In the case we consider, the widths $\Gamma_{j}$ arise because of quantum tunneling through a potential-energy barrier. We seek a semiclassical formula for this width-weighted spectrum - a formula analogous to periodic-orbit or closed-orbit formulas mentioned previously.

Why might a semiclassical representation of this widthweighted spectrum be of interest? Periodic-orbit and closed-orbit representations focus not on individual quantum states, but on the large-scale structure of the spec- trum. When we examine quantum processes whose rates are governed by tunneling (such as some chemical reaction rates, some nuclear decay rates, or some conductance processes in microstructures), it might be difficult to find the tunneling rate for each individual quasibound state. In such cases it may be useful to have available a simple representation of the averaged or large-scale structure of the tunneling rate. Thus we pose the following questions. Is there a semiclassical representation of the width-weighted spectrum $D \Gamma(E)$ ? What kind of paths are needed to represent it? How are those paths weighted?

These questions will be answered in this Letter for a system with a regular spectrum. Specifically, we consider the case of the hydrogen atom subjected to an external electric field. (However, many aspects of our derivation are valid for other regular systems, and also for spectra weighted in other ways.)

The hydrogen atom in an electric field is a physical system which permits a detailed study of the tunneling through a dynamical barrier. The Hamiltonian is (using atomic units $\hbar=1, e=1, m_{e}=1$ )

$$
H=\frac{\mathbf{p}^{2}}{2}-\frac{1}{\left(\rho^{2}+z^{2}\right)^{1 / 2}}+F z,
$$

where $F$ is the strength of the applied electric field. We consider the cylindrically symmetric states, $L_{z}=$ $m \hbar=0$. It is convenient to use scaled variables, $w=$ $F^{-1 / 4}, \mathbf{q} \rightarrow w^{-2} \mathbf{q}, \mathbf{p} \rightarrow w \mathbf{p}, \varepsilon=E / F^{1 / 2}$. The potential energy has a saddle point at $z_{s}=-1, \varepsilon=-2$. For energies below this saddle energy, the classical motion is bounded and the quantum spectrum is quasidiscrete, with states of long lifetime and quite sharply defined energy. High-Rydberg states of the pure Coulomb field are split into regular Stark manifolds. For energies above the saddle, the classical motion is unbounded if the electron is ejected from the atom in a "downhill" direction, and bounded if it leaves the atom going "uphill." A critical ejection angle $\theta_{c}(\varepsilon)=\arccos \left(1-\varepsilon^{2} / 2\right)$ separates these two motions. Accordingly, above the saddle, the quantum spectrum consists of quasidiscrete levels superposed 
on a smoothly rising continuum. The higher-energy (uphill) quasidiscrete levels of each manifold survive, while the lower-energy (downhill) levels in each manifold are broadened into the smooth continuum [5].

The Stark Hamiltonian (2) is integrable, and even separable in semiparabolic coordinates $(u, v)$, and the corresponding effective Hamiltonians are

$$
\begin{aligned}
& H_{u}=p_{u}^{2} / 2-\varepsilon u^{2}+u^{4} / 2=(1+\beta), \\
& H_{v}=p_{v}^{2} / 2-\varepsilon v^{2}-v^{4} / 2=(1-\beta) .
\end{aligned}
$$

Motion in the $u$ coordinate is always bound, but for $\varepsilon<0$ there is an effective (dynamical) barrier in the $v$ motion, which allows the possibility of tunneling. The classical $v$ motion is bound for $\varepsilon<-2$, and unbound for $\varepsilon \geq 0$. Between the saddle and the zero-field ionization threshold, i.e., for $-2 \leq \varepsilon \leq 0$, the $v$ motion is bound if and only if $\beta \geq \beta_{c}(\varepsilon)=1-\varepsilon^{2} / 2$. The separation constant $\beta$ is related to the angle of ejection of the electron from the atom by $\beta=\cos \theta$.

Accordingly, at each $(\varepsilon, w)$, the bound trajectories form a one-parameter family of tori with $\beta_{c}(\varepsilon) \leq \beta \leq 1$. The trajectories can therefore also be labeled by their action variables, $J_{u}(\varepsilon, \beta ; w), J_{v}(\varepsilon, \beta ; w)$. Quantization of these action variables identifies "eigentrajectories" or "eigentori," labeled by quantum numbers $\mathbf{n}=\left(n_{u}, n_{v}\right)$ (for more details see [6]). Standard semiclassical formulas [7], including tunneling near the top of a quadratic barrier in the $v$ coordinate, lead to quantization conditions for the energies and widths of the quasibound states:

$$
\begin{aligned}
J_{u}(\varepsilon, \beta ; w) & =w \tilde{J}_{u}(\varepsilon, \beta)=2 \pi \hbar\left(n_{u}+\frac{1}{2}\right), \\
J_{v}(\varepsilon, \beta ; w) & =w \tilde{J}_{v}(\varepsilon, \beta) \\
& =2 \pi \hbar\left(n_{v}+\frac{1}{2}\right)-\frac{i}{2} \hbar e^{-K / \hbar}-\delta .
\end{aligned}
$$

Quantities with a tilde are defined here as scaled quantities, for example, $\tilde{J}_{u}, \tilde{J}_{v}$ are the scaled actions. $K$ is the action integral for a cycle of underbarrier motion in the $v$ coordinate, $n_{u}=0,1,2, \ldots$ and $n_{v}=0,1,2, \ldots$ are integers which define the parabolic states and $\delta=\arg [\Gamma(1 / 2+$ $i K / 2 \hbar \pi)]-(K / 2 \hbar \pi)[\log (K / 2 \hbar \pi)]+K / \hbar \pi$ is the parabolic-barrier correction. This correction is often small, but in our case it is needed in order to avoid certain logarithmic singularities: near the top of the barrier (i.e., above the saddle and $\beta \approx \beta_{c}$ ) the derivatives $\partial J_{v} / \partial \varepsilon$ and $\partial J_{v} / \partial \beta$ diverge. However, the corresponding derivatives of $J_{v}+\delta$ are finite.

If the widths are not too large, they can be determined from Eq. (4) by the formula

$$
\Gamma_{\mathbf{n}}=\frac{\hbar \frac{\partial J_{u}}{\partial \beta}}{\frac{\partial\left(J_{u}, J_{v}\right)}{\partial(E, \beta)}} e^{-K / \hbar}=\frac{\frac{\partial \tilde{J}_{u}}{\partial \beta}}{w^{3} \frac{\partial\left(\tilde{J}_{u}, \tilde{J}_{v}\right)}{\partial(\varepsilon, \beta)}} e^{-w \tilde{K}},
$$

where $\partial\left(J_{u}, J_{v}\right) / \partial(E, \beta)$ is the Jacobian of the transformation from action variables $\left(J_{u}, J_{v}\right)$ to the conserved quantities $(E, \beta)$. In a one-dimensional system, the cor- responding formula for $\Gamma$ is $\hbar$ times the vibrational frequency times an exponential factor. Our system is separable, and tunneling is only in the $v$ coordinate, but it is not equivalent to a one-dimensional system. The tunneling terms make $E$ and $\beta$ complex in such a way that $J_{u}$ stays real. Therefore the preexponential factor in Eq. (5) involves both $J_{u}$ and $J_{v}$.

From these quantization conditions (4), we may construct a width-weighted spectrum, Eq. (1), holding $F$ fixed and finding $\left(E_{j}, \Gamma_{j}\right)$. The scaled spectrum is obtained by fixing $\varepsilon=E w^{2}$ and finding the corresponding (real) values of $w_{j}$; then

$$
D \Gamma=\sum_{\mathbf{n}} \Gamma_{\mathbf{n}} \delta\left(E-E_{\mathbf{n}}\right)=\sum_{\mathbf{n}} \frac{\tilde{\Gamma}_{\mathbf{n}}}{(-2 \varepsilon)} \delta\left(w-w_{\mathbf{n}}\right),
$$

where $\tilde{\Gamma}_{\mathbf{n}}=\Gamma_{\mathbf{n}} w^{3}$. In this calculation, we include all resonances which lie below the effective barrier in the $v$ coordinate; thus we include all quasidiscrete states, but we do not include above-barrier resonances, which may be so broad that they might better be regarded as background continuum.

Figure 1 shows an example of a scaled quasidiscrete spectrum for a scaled energy $(\varepsilon=-1.5)$ well above the saddle (at this scaled energy $\beta_{c}=-0.125, \theta_{c} \simeq$ $97.181^{\circ}$ ). Each point of this figure marks the width $\log \Gamma_{n}$ plotted against its quantized $w_{n}$ [8]. For example, at principal quantum number $n=n_{u}+n_{v}+1=9$, we see states with $u, v$ quantum numbers from $\left(n_{u}=8, n_{v}=\right.$ 0 ) (the most "uphill" and longest-lived state of the group) to $\left(n_{u}=3, n_{v}=5\right)$ (the most "downhill" and shortestlived state of the group). In this family, states with $n_{v} \geq$ 6 correspond to trajectories that go over the effective barrier, so we do not include them. The other states labeled in Fig. 1 are for $n=22$; in this family, $n_{v}$ can be as large as 13 before the trajectory goes over the barrier.

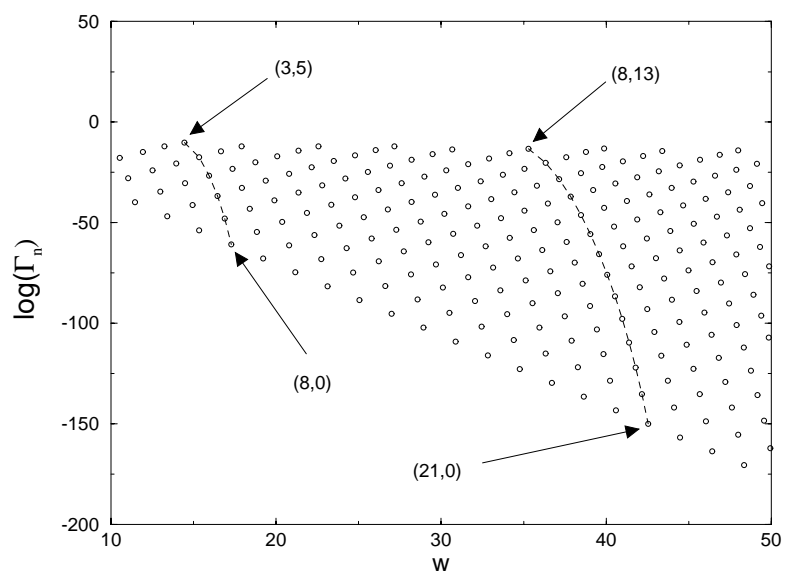

FIG. 1. Semiquantal result of $\log \Gamma_{\mathbf{n}}$ vs $w$ for the scaled energy $\varepsilon=-1.5$. The parabolic quantum numbers $\left(n_{u}, n_{v}\right)$ are labeled for the most "downhill" $(3,5),(8,13)$ and for the most "uphill" $(8,0),(21,0)$ states for the principal quantum numbers $n=9$ and $n=22$. 


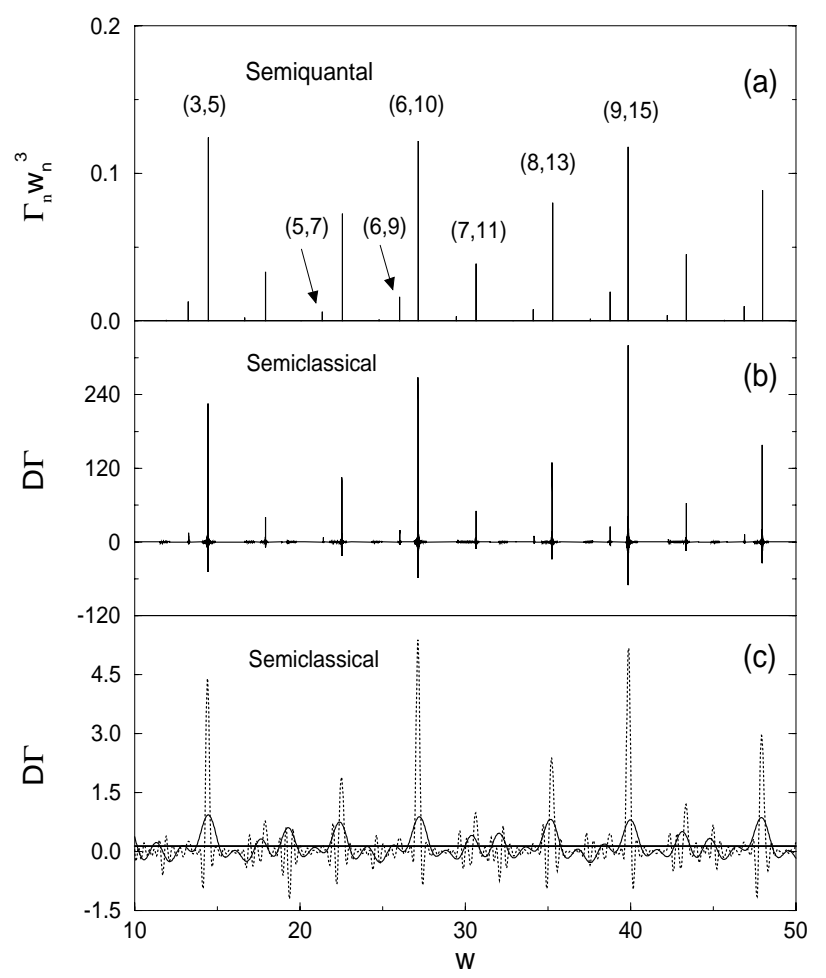

FIG. 2. Comparison between (a) semiquantal and (b), (c) semiclassical results for the width-weighted spectrum at scaled energy $\varepsilon=-1.5$. In the semiquantal result, some widths are labeled by the quantum numbers $\left(n_{u}, n_{v}\right)$. The straight line in (c) is the result from Eq. (7) for $M_{u}=M_{v}=0$. The solid line with few oscillations is the result from Eq. (7) taking the sum until $\left|M_{u}\right|=\left|M_{v}\right|=1$, and the dotted line is the result by the summation until $\left|M_{u}\right|=\left|M_{v}\right|=5$. (b) Is the result for $\left|M_{u}\right|=300,\left|M_{v}\right|=200[9]$.

Each state has also a quantized value of $\beta_{n}$; those states with the largest width (i.e., larger $n_{v}$ ) have $\beta_{n}$ closer to $\beta_{c}$. Such states are the most important in a widthweighted spectrum.

In Fig. 2(a), $\tilde{\Gamma}_{\mathbf{n}}$ is plotted as a function of $w$. The result looks like a quasiperiodic sequence of widths vs $w$. This is what we define as a width-weighted scaled spectrum. Such results of Eq. (5) are what could be regarded as "quantum results" [10]. More precisely they should be called "semiquantal": they use semiclassical approximations at certain points, but the formulas focus on energies and widths of individual states. The formulas developed below we call "semiclassical": they will be the analogs of the formulas of periodic-orbit theory or closed-orbit theory, which give simple representations of the large-scale structure of the spectrum.

Let us now write the semiclassical formula for $D \Gamma$. This formula involves properties of the "extremal torus," or "last surviving torus" at each scaled energy. As explained above, the motion is oscillatory in $v$ if $\beta>\beta_{c}(\varepsilon)$. When $\beta=\beta_{c}(\varepsilon)$, the $v$ motion ascends the dynamical barrier and hangs at the top, and we call the associated torus the extremal torus. For smaller $\beta$, the particle goes over the dynamical barrier and escapes (see Fig. 3).

Action variables of the extremal torus are well defined, $\hat{J}_{u}=\tilde{J}_{u}\left(\varepsilon, \beta_{c}\right), \hat{J}_{v}=\tilde{J}_{v}\left(\varepsilon, \beta_{c}\right)$. Derivatives of $\tilde{J}_{v}(\varepsilon, \beta)$ diverge as $\beta \rightarrow \beta_{c}(\varepsilon)$, but in the formulas below these divergences will be canceled by corresponding divergences in $\delta$.

The semiclassical formula is [11]

$$
D \Gamma=\sum_{M_{u}, M_{v}} D_{M_{u}, M_{v}} e^{i w\left[M_{u} \hat{J}_{u}+M_{v} \hat{J}_{v}\right]},
$$

where

$$
D_{M_{u}, M_{v}}=\frac{1}{4 \pi^{2}} \frac{(-)^{M_{u}+M_{v}}}{\left[\hat{\tau}_{K} / \hat{\tau}_{u}-i\left(M_{u}-M_{v} \hat{\tau}_{v} / \hat{\tau}_{u}\right)\right]} .
$$

All quantities here must be evaluated at $\beta=\beta_{c}$. The sum over $M_{u}$ and $M_{v}$ includes all positive and negative integers, including zero, so the result is indeed a quasiperiodic function of $w$ : it oscillates as a function of $w$ with fundamental frequencies $\hat{J}_{u}$ and $\hat{J}_{v}$, and with all multiples and combinations thereof. Each oscillation has amplitude $D_{M_{u}, M_{v}}$, which is a function of the "canonical periods"

$$
\begin{aligned}
& \hat{\tau}_{u}=2 \partial \tilde{J}_{u} / \partial \beta, \quad \hat{\tau}_{K}=2 \partial \tilde{K} / \partial \beta, \\
& \hat{\tau}_{v}=-2 \partial\left(\tilde{J}_{v}+\delta / w\right) / \partial \beta,
\end{aligned}
$$

all of which are evaluated at $\beta=\beta_{c}$.

Figure 2(c) shows the result of taking more and more terms in the summation of Eq. (7). The straight line is

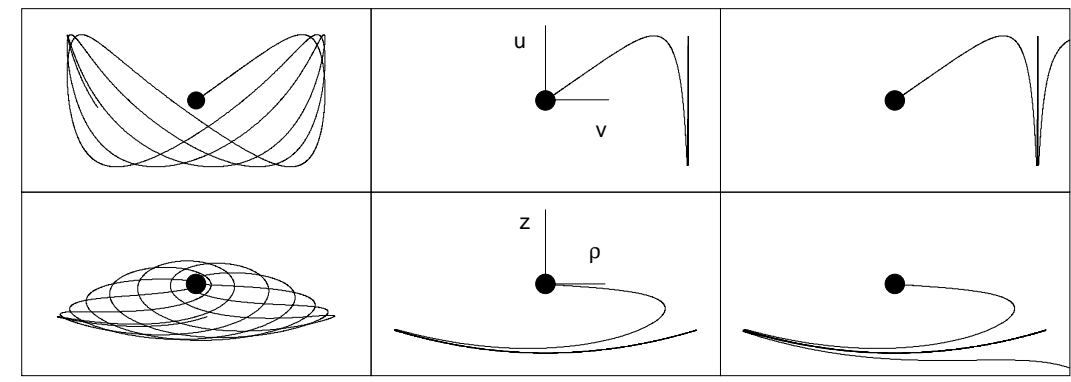

FIG. 3. Electron orbits in $u v$ (upper) or $z \rho$ (lower). Left: For ejection angle less than the critical angle $\theta_{c}$ the orbit remains bound. Right: For larger ejection angles it goes over the effective barrier and escapes. Center: At the critical angle the orbit approaches an unstable periodic orbit - these orbits lie on the extremal torus. 


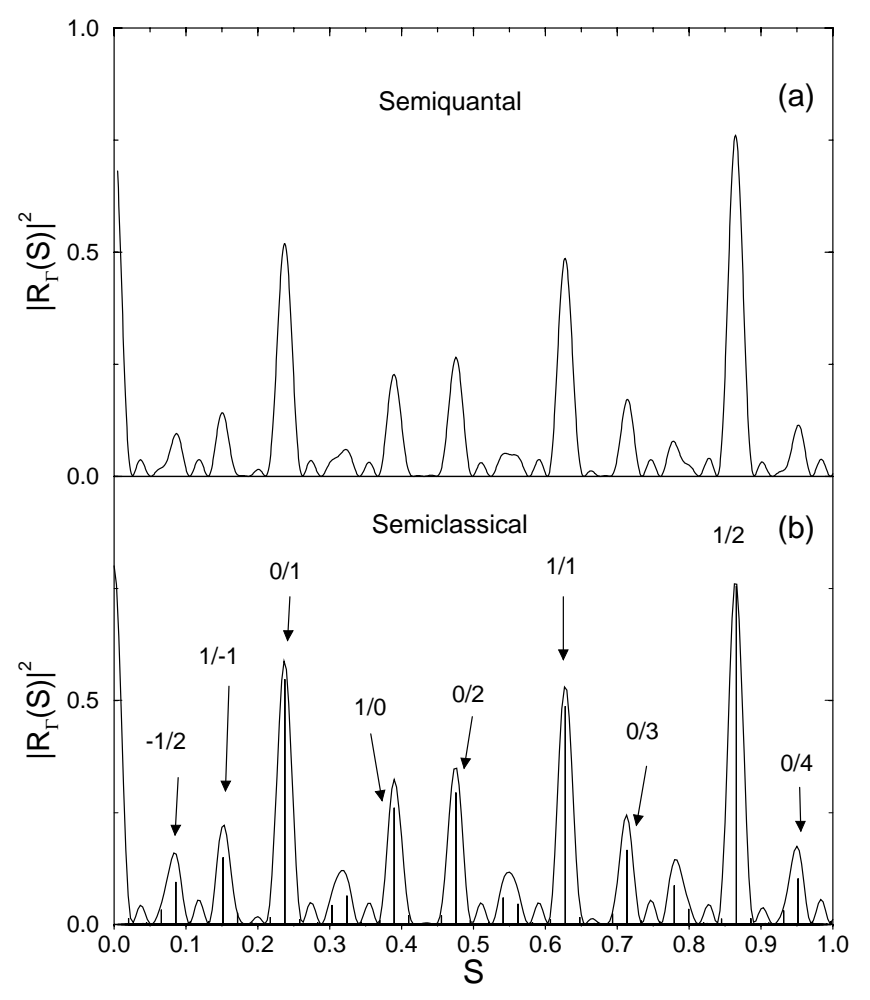

FIG. 4. Comparison between (a) semiquantal and (b) semiclassical results for the absolute square of the Fourier transform at the scaled energy $\varepsilon=-1.5$. Peaks in the semiclassical result are located at actions $S_{M_{u}, M_{v}}$, and they are labeled by the cycle numbers $\left(M_{v} / M_{u}\right)$. The needles under these peaks correspond to the values of $\left|D_{M_{u}, M_{v}}\right|^{2}$.

the result for $M_{u}=M_{v}=0$. The solid line with few oscillations is the result of taking eight more terms in the sum (i.e., $\left|M_{u}\right|=\left|M_{v}\right|=1$ ), and the dotted line is for summation until $\left|M_{u}\right|=\left|M_{v}\right|=5$. Figures 2(a) and 2(b) compare the semiclassical numerical results from Eq. (7) (with $\left|M_{u}\right|^{\max }=300,\left|M_{v}\right|^{\max }=200$ ) with the semiquantal results from Eq. (5) for a scaled energy $\varepsilon=$ -1.5 . The positions of the maxima for a given state $w_{\mathbf{n}}$ are in good agreement and also the overall behavior of the peaks is the same. [Of course, the Fourier sum (7) gives the expected Gibbs' phenomenon.]

Another way to analyze and understand the widthweighted spectrum is to look at its Fourier transform,

$$
R_{\Gamma}(S)=\int \exp (-i 2 \pi S w) D \Gamma(w) d w
$$

$\left|R_{\Gamma}(S)\right|^{2}$ should have peaks located at values of scaled action $S_{M_{u}, M_{v}}=\left(M_{u} \hat{J}_{u}+M_{v} \hat{J}_{v}\right) / 2 \pi$, with heights proportional to $\left|D_{M_{u}, M_{v}}\right|^{2}$. This is exactly what we see in Figs. 4(a) and 4(b), where the (normalized) absolute square of the Fourier transform is plotted as a function of the variable $S$. For this scaled energy, the value of the scaled actions of the extremal torus are $\widehat{J}_{u} / 2 \pi \simeq 0.23796$ and $\hat{J}_{v} / 2 \pi \simeq 0.38985$.
With the help of the semiclassical representation of the the width-weighted spectrum, each peak in the Fourier transform [see Fig. 4(b)] can be identified with a given irreducible loop on the extremal torus, having $M_{u} u$-cycles and $M_{v} v$-cycles. We emphasize that these loops do not in general correspond to closed orbits or to periodic orbits. For example, we see a large $1 / 1$ peak, but the frequencies of the $u$ and $v$ motions are never equal, and no $(1 / 1)$ periodic or closed orbit exists.

The needles under the peaks in Fig. 4(b) correspond to the values of $\left|D_{M_{u}, M_{v}}\right|^{2}$. The height of the maxima in the Fourier transform of the semiclassical results depends strongly on the parabolic correction $\delta$; this happens because $\delta$ is only a small correction to $\hat{J}_{v}$, but its derivative is a substantial part of $\hat{\tau}_{v}$ and therefore of $D_{M_{u}, M_{v}}$.

To conclude, in this Letter we define a width-weighted spectrum, and we give a semiclassical representation of it. As an example we consider the scaled spectrum of the hydrogen atom in an electric field. The Fourier transform of this scaled width-weighted spectrum gives peaks not at actions of periodic orbits, nor at actions of closed orbits, but at actions corresponding to fundamental loops of the extremal torus: The torus corresponding to limiting motion climbing to the top of the effective barrier.

This research was supported by NSF. M. W. B. thanks CAPES for financial support.

[1] M. C. Gutzwiller, J. Math. Phys. (N.Y.) 11, 1791 (1970).

[2] M. V. Berry and M. Tabor, Proc. R. Soc. London A 349, 101 (1976).

[3] M. L. Du and J. B. Delos, Phys. Rev. A 38, 1896 (1988); 38, 1913 (1988).

[4] S. C. Creagh and N. D. Whelan, Phys. Rev. Lett. 77, 4975 (1996); (to be published).

[5] V. Kondratovich and J. B. Delos, Phys. Rev. A 57, 4654 (1998).

[6] V. Kondratovich and J.B. Delos, Phys. Rev. A 56, R5 (1997).

[7] M.S. Child, Semiclassical Mechanics with Molecular Applications (Oxford University Press, New York, 1991).

[8] This is the width for decay by tunneling. These states can also decay by photon emission, but that is not of interest here.

[9] The scales of the vertical axes in Figs. 3(a)-3(c) are different because the Fourier representation "converges" to $\delta$ functions. They go to infinity, but their integrated areas are proportional to $\Gamma_{n} w_{n}^{3}$.

[10] We compared the results of Eqs. (4) and (5) with numerical quantum calculations [12]. We found agreement of positions and widths to within $0.03 \%$ and $1.5 \%$, respectively.

[11] Proof will be given in a future paper.

[12] G. Alvarez, R. G. Damburg, and H. J. Silverstone, Phys. Rev. A 44, 3060 (1991). 